\title{
Métodos computacionales para la creación artística
}

\author{
Sandra Rodríguez-Mondragón, Luis Jorge Soto-Walls, \\ Manuel Martín Clavé-Almeida, Oscar Herrera-Alcántara \\ Universidad Autónoma Metropolitana, Unidad Azcapotzalco, \\ Ciudad de México, México \\ \{sandra.rgz.mondragon, luissotowalls, mclavealmeida\}@gmail.com, \\ oha@correo.azc.uam.mx
}

\begin{abstract}
Resumen. Se presentan algunas formas de aplicar principios algorítmicos al desarrollo de obra artística con carácter digital. Como parte inicial se abordan las definiciones de algunos conceptos tales como obra de arte, arte digital, algoritmo y tiempo real; se describen cuatro métodos algorítmicos donde es posible incluir el concepto de tiempo real. Se abordan algunas formas de expresión artística desarrolladas por medio de algoritmos como los fractales, el arte sonoro y el data mining. Finalmente se describe cómo se pueden implementar las metodologías del diseño de algoritmos descritas al desarrollo de arte digital, donde interviene el tiempo real como variable independiente para la creación artística y se presentan algunos desarrollos de producción artística con IA.
\end{abstract}

Palabras clave: arte, arte digital, tiempo real, algoritmo, fractal, data mining.

\section{Computational Methods for Artistic Creation}

\begin{abstract}
Some ways of applying algorithmic principles to the development of artistic work with digital character are presented. As an initial part, the definitions of some concepts such as work of art, digital art, algorithm and real time are addressed; Four algorithmic methods are described where it is possible to include the concept of real time. Some forms of artistic expression developed through algorithms such as fractals, sound art and data mining are also tackled. Finally, we describe how the methodologies of the described algorithm design can be implemented, to the development of digital art where real time intervenes as an independent variable for artistic creation and are presented some developments of artistic production with AI.
\end{abstract}

Keywords: art, digital art, real time, algorithm, fractal, data mining.

\section{Definiciones}

\subsection{Obra de arte}

Con objeto de lograr definir el concepto de obra de arte, revisaremos algunas afirmaciones: Julio Domínguez define la obra de arte como "la manifestación concreta 
de genio creativo del hombre, cuando modifica o reorganiza su entorno para impactar en los sentimientos de sus semejantes" [1].

Por su parte Ricardo Padilla, nos dice que "la obra plástica es de distintas clases y texturas: papel, tela, madera, mármol, bronce o cualquier otro material susceptible de transformarse como la arcilla o el acrílico, y son tanto bidimensionales (dibujos, cuadros, grabados, litografías, etc.) como tridimensionales (esculturas, estatuas, obras arquitectónicas, monumentos, edificios, etc.), independientemente de su género (figurativo o abstracto) y de su finalidad" [2].

Así, Laura Fernández afirma que "en la obra plástica van a incidir dos partes, la formal... y la conceptual... existen elementos que le son comunes a todas las obras del arte que son configuradores...: líneas, áreas, volumen, hay elementos diferenciadores: color, calor tonal, textura y otras leyes que componen las obras de arte son las estéticas, en las cuales se encuentran: proporción, equilibrio, ritmo o énfasis" [3]. Y finalmente Terry Smith afirma que "antes que cualquier otra cosa, las obras de arte son testimonio de cada una de estas contemporaneidades: el mero hecho de llegar a ser en y por sí mismas, su existencia en un mundo repleto de otros y su persistencia en distintos mundos determinados por la repetición y la diferencia" [4].

Así la obra de arte nos permite expresar un concepto y éste se puede materializar bidimensional o tridimensionalmente, dando pie al objeto artístico que es perceptible por medio de los sentidos de la vista (visión) y del tacto; de la misma manera hay manifestaciones artísticas que se pueden percibir por medio de los otros sentidos eloído (audición), el olfato, el gusto.

\subsection{Generación de la obra artística}

Los factores que intervienen en el momento de la producción de obra artística pueden ser múltiples, sin embargo, existen aspectos básicos que se deben abordar. A lo largo de la historia ha existido la obra artística y los artistas, cada uno en su tiempo, se han valido de la tecnología para la generación de su obra, algunos han innovado y otros han explotado el uso de herramientas o materiales; así, por ejemplo, las primeras esculturas realizadas en mármol se labraron con cincel que era la tecnología disponible en ese momento histórico. Actualmente existen múltiples herramientas digitales que permiten al artista lograr la generación de obra de vanguardia.

En su momento el diseño gráfico dio un salto al diseño asistido por computadora y en la actualidad, las computadoras son herramientas fundamentales de esta disciplina. Los elementos para la generación de una obra artística escultórica (por ejemplo) son básicamente tres: el diseño (concepto y forma), el material y la tecnología a emplear en el desarrollo de la obra, en ello el escultor al realizar un diseño lo visualiza de forma ideal, después decide en qué material se producirá y finalmente por medio de herramientas logrará que el material adquiera la forma proyectada. Así, el diseño regirá sobre el material y la tecnología, tomando como principio que la manifestación artística es preponderante sobre las tecnologías que permiten su producción (ver Fig. 1). 


\subsection{Arte digital}

Estamos inmersos en un medio digital -tecnológico- en nuestra vida cotidiana, donde las nuevas tecnologías de comunicación e información (TIC's) se manifiestan en el arte, por ello surgen nuevos modos de expresión basados en las TIC's que potencializan los modos de hacer arte. En ello surge el concepto de arte digital como uno dentro de las nuevas artes.

De esta manera, el vínculo entre el arte y la tecnología se remonta a los años cincuenta, cuando un reducido grupo de artistas, de hecho, los pioneros del arte digital, empezó a utilizar la computadora para crear lo que se llamó las primeras pinturas electrónicas; es decir, arte hecho a través de programas informáticos comerciales para la edición de imagen o creación de dibujos. De acuerdo con Laura Regil, este género es producto de la creatividad artística y la mezcla de tecnologías digitales, ciberespacio y vida artificial, constituyéndose en un universo original de estética, espacio y tiempo. A su vez, las actuales manifestaciones artísticas experimentan y amplían tanto las posibilidades expresivas y de divulgación como las de la participación y apreciación de los espectadores [5].

Dentro de las "nuevas artes" (ver Fig. 2) podemos incluir a la producción artística que se genera a partir de las nuevas tecnologías y por medio de conceptos matemáticos que sugieren el arte a partir de esquemas susceptibles de reproducción controlada. Las nuevas artes provienen de un proceso creativo que se beneficia de la tecnología para lograr formas y efectos que, sin ella, simplemente no podrían realizarse, quedando en arte conceptual. Surgen así formas innovadoras de alta calidad y estructuras resueltas matemáticamente de forma muy precisa, que, si bien pueden generarse por medio de otros procesos, con la tecnología son resueltas eficientemente. Este tipo de obra es producto de conjugar diversos medios de expresión, vía la tecnología, y obtener un concepto más rico para todos los sentidos del ser humano, de esta manera se integra la música con la imagen, el arte que se difunde en Internet, el dibujo y el modelado computarizado, entre otros medios que combinados logran un resultado expresivo muy diverso.

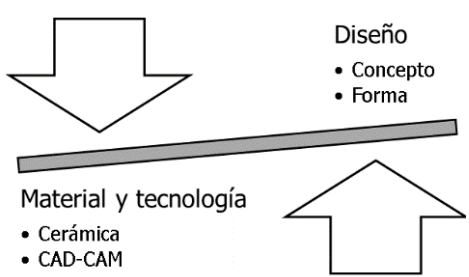

Fig. 1. Producción de obra artística.

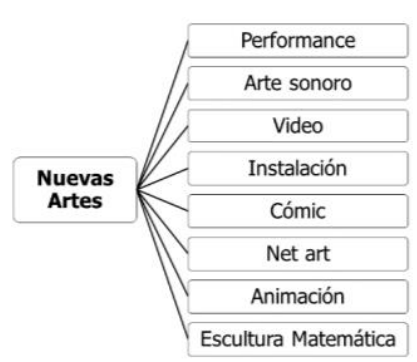

Fig. 2. Las nuevas artes, basado en las definiciones de Irma Escobar [6].

A modo de ejemplo de las manifestaciones artísticas que emplean principios matemáticos tenemos la clasificación que hace Ricardo Zalaya, de la escultura 
matemática, ello nos permite visualizar el potencial de este tipo de formas de expresión (ver Fig. 3) [7].

\subsection{Algoritmo}

Como dato histórico, tenemos que la palabra algoritmo trae su origen en el nombre del matemático persa "Mohamed ibn Musaal Khwarizmi" (825 d.C.). Su apellido fue traducido al latín como algorismus y posteriormente pasó al españolcomo Algoritmo. Khwarizmi fue bibliotecario en la corte del califa al- Mamun y astrónomo en el observatorio de Bagdad. Sus trabajos de álgebra, aritmética y tablas astronómicas adelantaron enormemente el pensamiento matemático y fue el primero en utilizar la expresión al-yabr (de la que procede la palabra álgebra). Su trabajo con los algoritmos introdujo el método de cálculo utilizando la numeración arábiga y la notación decimal [8].

Por otro lado, Francisco Pinares et. al, afirman que "para implementar la solución de un problema mediante el uso de una computadora, es necesario establecer una serie de pasos que permitan resolver el problema, a este conjunto de pasos se le denomina algoritmo, el cual debe tener como característica final la posibilidad de transcribirlo fácilmente a un lenguaje de programación, para esto se utilizan herramientas de programación, las cuales son métodos que permiten la elaboración de algoritmos escritos en un lenguaje entendible" [9]. Gustavo López et. al, lo definen como "un conjunto de pasos que, ejecutados de la manera correcta, permiten obtener un resultado (en un tiempo acotado)" [10].

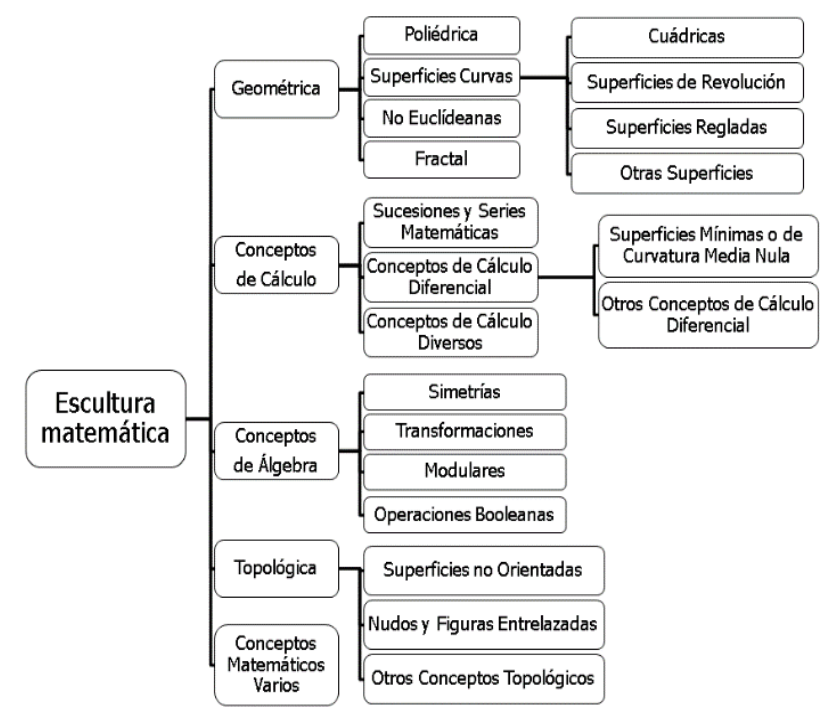

Fig. 3. Clasificación de escultura matemática. Basado en la propuesta de Zalaya.

Pueden existir varios algoritmos para resolver un mismo problema. Cuando se estudian los algoritmos es importante analizar tanto su diseño como su eficiencia. Se 
puede complementar la definición con las tres características que indica Pinales [9], además de la facilidad para transcribirlo: preciso (debe indicar el orden en el cual debe realizarse cada uno de los pasos que conducen a la solución del problema), definido (el resultado nunca debe cambiar bajo las mismas condiciones del problema) y finito (no se debe caer en repeticiones de procesos de manera innecesaria; deberá terminar en algún momento).

Así un algoritmo es un método para resolver un problema. En lo que a esta investigación refiere, citaremos algunos métodos computarizados con base en algoritmos para producir obra artística, aplicando el concepto de tiempo real. Donde la metodología que aplica algoritmos es una metodología que resuelve problemas por medio de una secuencia de pasos precisa, definida y finita.

Por otro lado, otra cualidad de los algoritmos es el lenguaje en que se pueden representar y ello lo retomamos de la descripción que hace Matías García [11], donde indica básicamente tres:

- Lenguaje natural, es el lenguaje común (coloquial).

- Lenguaje estructurado, con reglas sintaxis y semántica definidas, donde se puede usar pseudocódigo o lenguaje universal de programación; o de código que implica el uso de un programa en específico.

- Lenguaje simbólico que se puede desarrollar principalmente por medio de diagramas de flujo.

Así en lo que a algoritmo se refiere, de acuerdo con Ramírez, se deben considerar sus tres partes [12]: (1) Entrada: Información dada al algoritmo. (2) Proceso: Operaciones o cálculos necesarios para encontrar la solución del problema. (3) Salida: Respuestas dadas por el algoritmo o resultados finales de los procesos realizados.

De acuerdo con Carlos Balderrama [13], existen dos tipos de algoritmos: cualitativos que son aquellos en los que se describen los pasos utilizando palabras; y cuantitativos que son aquellos en los que se utilizan cálculos numéricos para definir los pasos del proceso.

\subsection{Tiempo real}

En el ámbito de la tecnología, el tiempo real se aplica a sistemas digitales en los que se produce una interacción en que se corresponde el tiempo interno del sistema con el tiempo externo o del ambiente. Por lo tanto, en un sistema en tiempo real se produce una interacción con el mundo real, entendido como un proceso físico, en el que se emiten respuestas correctas siguiendo las restricciones temporales. Algunos ejemplos de áreas concretas en que se puede utilizar este término son determinadas telecomunicaciones como las emisiones televisivas en directo o en meteorología, cuando un dispositivo indica la temperatura existente en el momento actual.

Los sistemas en tiempo real se utilizan ampliamente el día de hoy, tanto en la industria aérea, automotriz, ferroviaria, en el sector militar, en la medicina, en los electrodomésticos, prácticamente en cualquier aplicación que utilice software.

De la misma forma Alejandro Veigia [13], define a continuación un sistema de tiempo real como "un sistema que debe satisfacer restricciones explícitas en el tiempo 
de respuesta o arriesgarse a severas consecuencias, incluida la falla. Por tanto, un sistema de tiempo real es un sistema que responde a un estímulo externo dentro de un tiempo especificado. Su eficiencia no solo depende de la exactitud de los resultados de cómputo, sino también del momento en que los entrega. La predictibilidad es su característica principal. A diferencia de los sistemas tradicionales, que tienden a distribuir en forma equitativa los recursos disponibles entre las diferentes tareas a ejecutar, los sistemas de tiempo real deben asegurar la distribución de recursos de tal forma que se cumplan los requerimientos de tiempo". De la anterior afirmación cabe mencionar que la definición de tiempo real depende del tipo de aplicación que se dé al concepto.

\section{Métodos algorítmicos}

Si queremos producir una obra artística basada en software, primero tendremos que atravesar un proceso de diseño, conceptualización, escritura, compilación, depuración de errores, etc. Este proceso se encuentra mucho más relacionado con la ingeniería que con las artes plásticas.

De acuerdo con Balderrama [14], algunas de las herramientas utilizadas comúnmente para diseñar algoritmos son: seudocódigo, diagrama de flujo, diagramas Nassi/Shneiderman-S (Chapin), método Jackson, método Bertini, método Tabourier, método Warnier, entre otros (ver fig. 4).

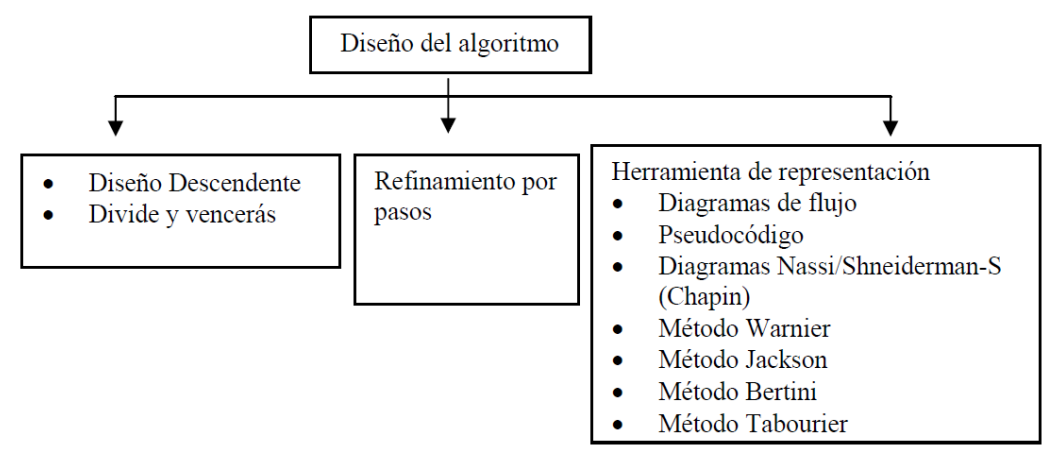

Fig. 4. Diseño de algoritmo.

Un computador no tiene capacidad para solucionar problemas más que cuando se le proporciona los sucesivos pasos a realizar, estos pasos indican las instrucciones a ejecutar por la máquina y se denomina algoritmo. Normalmente los pasos diseñados en un primer esbozo del algoritmo son incompletos e indican sólo unos pocos pasos, tras esta primera descripción estos se amplían en una descripción más detallada con pasos específicos; dicho proceso se denomina refinamiento del algoritmo. Las características de un buen algoritmo son:

- Debe tener un punto particular de inicio.

- Debe ser definido, no debe permitir dobles interpretaciones. 
- Debe ser general, es decir, soportar la mayoría de las variantes que se puedan presentar en la definición del problema.

- Debe ser finito en tamaño y tiempo de ejecución [14].

En lo que refiere a la generación artís tica, es posible aplicar el principio algorítmico que se desee, ello dependerá del concepto de la obra, el material o forma en que se representará y por supuesto los recursos con que se cuente para su producción. A continuación, menciono algunos de los métodos que ya se han usado para tal fin, sin que ello se contemple como una compilación exhaustiva, pero si con carácter didáctico; cabe mencionar que en los casos que se aplica el concepto de tiempo real generalmente nos referimos a eventos en los que el medio de expresión es el sonido, aunque también podemos hablar de visualización (video, foto, imagen digital, etc.).

\subsection{Composición algorítmica}

El concepto de composición algorítmica se refiere al método de creación musical por medio de procesos formales o algoritmos. Es un método para la creación de música no lineal, es decir cuando los eventos sonoros no están predeterminados hasta el momento en que ocurren [14].

La composición algorítmica se puede desarrollar por medio de algoritmos genéticos, gramáticas formales, autómatas celulares y cadenas de Markov; en este ensayo sólo se describen las antes mencionadas, pese a que se pueden aplicar otras.

\subsection{Algoritmos genéticos}

Retomando la definición de Fogel, son métodos adaptativos, generalmente usados en problemas de búsqueda y optimización de parámetros, basados en la reproducción sexual y en el principio de supervivencia del más apto [15]. Así, Goldberg afirma que "son algoritmos de búsqueda basados en la mecánica de selección natural y de la genética natural. Combinan la supervivencia del más apto entre estructuras de secuencias con un intercambio de información estructurado, aunque aleatorizado, para constituir así un algoritmo de búsqueda que tenga algo de las genialidades de las búsquedas humanas" [16].

Tabla 1. Operatividad de gramática formal [18].

\begin{tabular}{|c|c|c|c|c|}
\hline Gramática & Lenguaje & $\begin{array}{c}\text { Reglas de } \\
\text { Producción }\end{array}$ & $\begin{array}{l}\text { Si } \mu \rightarrow \varphi \text {, relación } \\
\text { entre }|\mu| \text { y }|\varphi|\end{array}$ & Solución \\
\hline Tipo-0 & Recursivas & Sin restricciones & & Máquinas de Turing \\
\hline Tipo-1 & Dependiente de contexto & $\alpha \mathrm{A} \beta \rightarrow \alpha \gamma \beta$ & $|\mu| \leq|\varphi|$ & $\begin{array}{l}\text { Autómatas lineales } \\
\text { acotados }\end{array}$ \\
\hline Tipo-2 & Independiente de contexto & $A \rightarrow \gamma$ & $|\mu|=1$ & Autómatas de pila \\
\hline Tipo-3 & Regular & $\begin{array}{l}A>\rightarrow a B \\
A \rightarrow a\end{array}$ & & $\begin{array}{l}\text { Autómatas finitos, } \\
\text { regulares }\end{array}$ \\
\hline
\end{tabular}

$\alpha \beta$ y $\gamma$ son cadenas de terminales y no terminales. La cadena $\gamma$ no puede ser la cadena vacía.

Para alcanzar la solución a un problema se parte de un conjunto inicial de individuos, llamado población, generado de manera aleatoria. Cada uno de estos individuos 
representa una posible solución al problema. Estos individuos evolucionarán tomando como base los esquemas propuestos por Darwin sobre la selección natural, y se adaptarán en mayor medida tras el paso de cada generación a la solución requerida [17].

\subsection{Gramáticas formales}

Una gramática formal consta de un conjunto finito de símbolos terminales (las palabras en un lenguaje formal), un conjunto finito de símbolos no terminales, un conjunto de reglas de producción con un lado izquierdo y otro derecho, y un símbolo inicial. Las reglas se aplican sustituyendo la parte de la izquierda por la parte de la derecha. Una derivación es una secuencia de aplicaciones de reglas. Cada gramática define el lenguaje formal de todas las sentencias que están formadas exclusivamente por los símbolos terminales a los que se puede llegar mediante derivación a partir del símbolo inicial.

Así, Gonzalo, describe cuatro tipos de gramáticas formales: tipo 0 (sin restricciones, recursivas), tipo 1 (dependientes de contexto), tipo 2 (independientes de contexto, libre de contexto) y tipo 3 (gramáticas regulares). Y también afirma que: todo lenguaje de tipo 3 es de tipo 2, todo lenguaje de tipo 2 es de tipo 1, y todo lenguaje de tipo 1 es de tipo 0 [18]. Lo anterior también, en lenguaje de programación, se puede representar como en el cuadro que se muestra a continuación en la tabla 1.

\subsection{Autómatas celulares}

Un autómata celular es un modelo matemático para un sistema dinámico, compuesto por un conjunto de celdas o células que adquieren distintos estados o valores. Estos estados son alterados de un instante a otro en unidades de tiempo discreto, es decir, que se puede cuantificar con valores enteros a intervalos regulares. De esta manera, este conjunto de células logra una evolución según una determinada expresión matemática, que es sensible a los estados de las células vecinas, la cual se le conoce como regla de transición local. El aspecto que más los caracteriza es su capacidad de lograr una serie de propiedades que surgen de la propia dinámica local a través del paso del tiempo y no desde un inicio, aplicándose a todo el sistema en general. Por lo que no es fácil analizar sus propiedades globales desde su comienzo, complejo por naturaleza, a no ser por vía de la simulación, partiendo de un estado o configuración inicial de células y cambiando en cada instante los estados de todas ellas de forma síncrona [19].

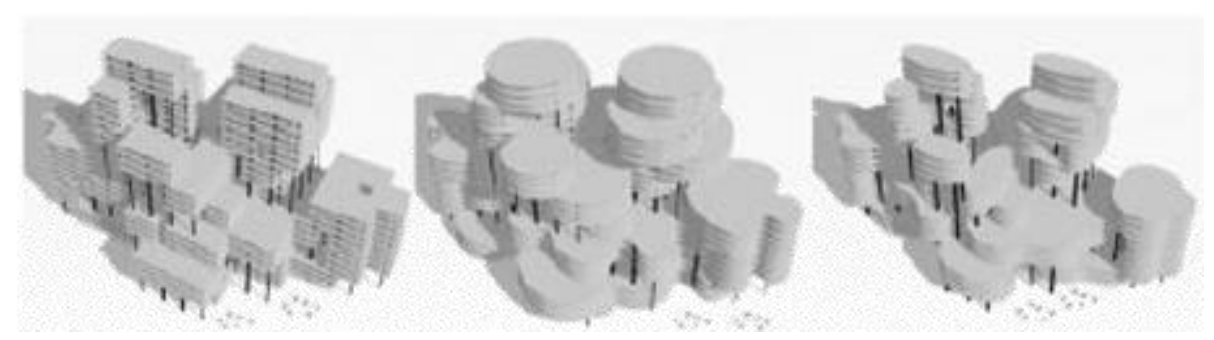

Fig. 5. Formas arquitectónicas básicas que surgen de un autómata celular. 
Algunas de las aplicaciones de autómatas celulares se dan en la arquitectura, bioinformática y la criptografía.

Arquitectura. La conexión que se hace con ésta es la capacidad de generar patrones o modelos y, de una forma organizada, estos modelos nos pueden sugerir formas arquitectónicas (ver Fig. 5).

La Bioinformática consiste en analizar, comprender y predecir procesos biológicos con la ayuda de herramientas computacionales. Puede ser vista como la disciplina que une dos ciencias: Biología y Computación (ver Fig. 6).

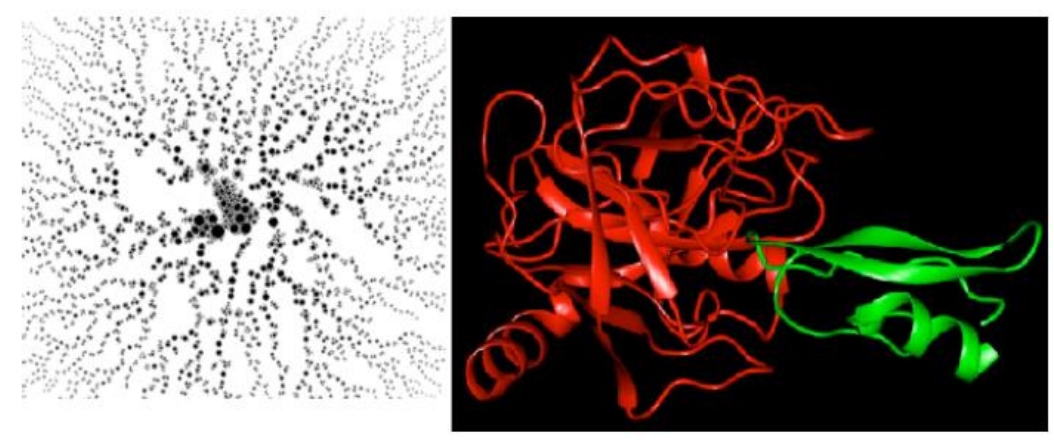

Fig. 6. Interacciones entre proteínas, tema común de estudio de la Bioinformática.

Por necesidades del ser humano, transmitir información de forma segura, surge la criptología (del griego cripto-oculto- y logos-tratado, ciencia), ésta se divide en dos ramas: criptografía, cuya tarea es cifrar la información a enviar, y criptoanálisis que se encarga de analizar técnicas y métodos para obtener la información cifrada (ver Fig. 7).

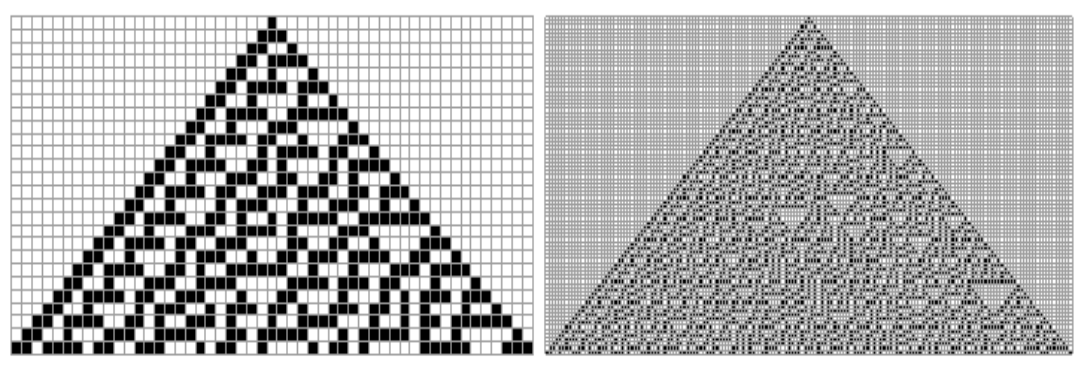

Fig. 7. Diagrama de evolución del autómata celular, regla 30 en 25 y 100 generaciones.

\section{Arte algorítmico}

A pesar de su aparente especificidad, la creación de imágenes de síntesis no difiere en lo fundamental del proceso creativo de las artes plásticas. Por lo que en ambos procesos de creación de imágenes (abstracciones visibles), a partir de modelos surgidos 
de la imaginación o la observación de sus creadores; a diferencia de cualquier otra técnica anterior, las técnicas digitales permiten además reproducir (y modificar) imágenes de cualquier origen de un modo sencillo, rápido y barato. Un orden visual renovado del que surge una nueva situación iconográfica.

\subsection{Fractal}

Todas las imágenes creadas y reproducidas por computadora son el resultado de una sucesión de algoritmos matemáticos. Un caso particular de imagen sintética son las imágenes fractales, figuras geométricas formadas por un número infinito de elementos de pequeñez infinita, contenidos en una superficie infinita que sólo se pueden ver, siguiendo determinados algoritmos, con la ayuda de computadoras (ver. Fig. 8).

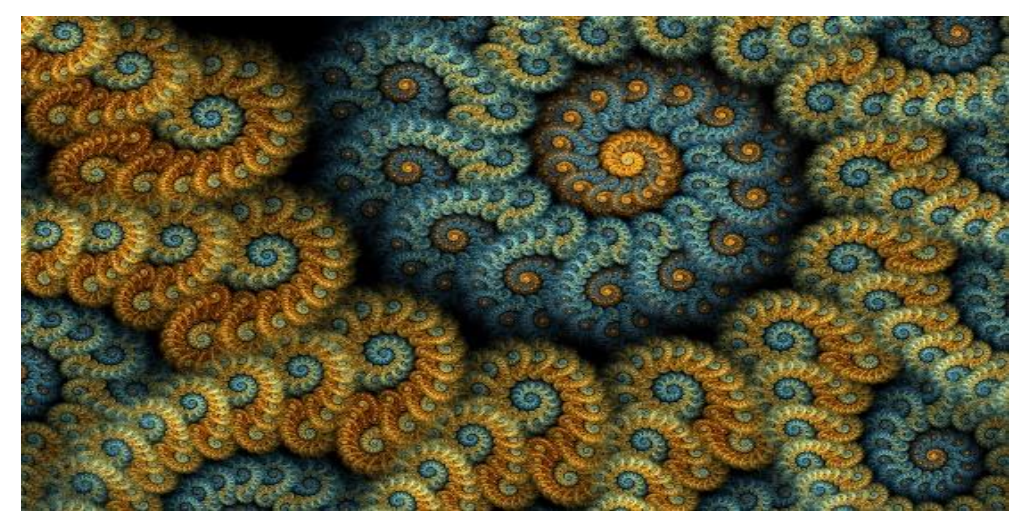

Fig. 8. Ejemplo de fractal desarrollado por computadora.

\subsection{Algoritmos en arte sonoro}

Con la ayuda de medios digitales y analógicos actuales e inspirándose en la tradición de pioneros del vídeo experimental como Nam June Paik y Woody y Steina Vasulka, Koolwijk y Prins compartieron sus conocimientos técnicos y con su proyecto Synchronator se lanzaron a resolver las dificultades involucradas en el registro de señales de vídeo y audio complejas, híbridas y distorsionadas. En directo, su performance audiovisual cuenta con dos proyecciones simultáneas y es consecuencia directa de su interés mutuo en convertir la imagen en sonido y viceversa, haciendo uso de los métodos que han desarrollado para conseguir que equipos de vídeo convencionales acepten señales analógicas, y a veces de audio y vídeo perturbados, como si fueran señales legítimas.

Regularmente cuando se trabaja con arte sonoro se hace uso de Procesadores Digitales de Señal (DSP), lo que permite captar el sonido y convertirlo a datos que se pueden procesar en un ordenador. Los procesadores DSP pueden realizar tareas en tiempo real, sin embargo, la definición de tiempo real depende del tipo de aplicación [20]. 


\subsection{Data mining o minería de datos (DM)}

Sonia Pighin la describe como la extracción de información en grandes bases de datos. Su nombre deriva de la analogía existente entre buscar dicha información valiosa y minar una montaña para encontrar un yacimiento de metales preciosos, ya que ambos procesos requieren examinar una inmensa cantidad de material o investigar inteligentemente hasta concretar la búsqueda.

Es un conjunto de tecnologías que ayuda a las empresas a enfocar sus objetivos sobre la información más importante de sus fuentes de datos. Las herramientas de DM pueden responder preguntas que generalmente demandan demasiado tiempo, encontrando información que ni un profesional experto podría hallar porque se encuentra fuera de sus expectativas. DM es en realidad, un proceso iterativo de descubrimiento de patrones y tendencias dentro de los datos, a través de métodos automáticos, manuales o más generalmente semiautomáticos, y que no serían necesariamente revelados por otros métodos tradicionales de análisis. Las herramientas de DM exploran las bases de datos en busca de patrones ocultos, permitiendo a partir de éstos, predecir las futuras tendencias y comportamientos de información nueva [21].

Una de las ventajas del DM, consiste en que el manejo de la información se puede realizar por medio de texto o de forma visual, algunas de las formas de visualizar los datos son: agrupación, estructuras jerárquicas, redes y panorámicas.

Con base en el DM, algunos artistas visuales como Ulises Carrión, Daniel Lara, Víctor Manuel Dávalos, Felipe Ehremberg, entre otros, han implementado el concepto de tiempo real o transmisión automática debido a que por medio de un DSP se captan las frecuencias en un concierto en vivo ya sea de música tradicional o electrónica.

\section{Conclusión}

Es complicado citar un método general para la resolución de problemas, en este caso para producir obra artística por medio de algoritmos aplicando la variable de tiempo real, la resolución de un problema es un proceso creativo donde el conocimiento, la habilidad y la experiencia tienen un papel importante, el proceder de manera sistemática ayuda a resolver cualquier problema y el diseño de algoritmos es una forma estructurada de sistematizar la forma de resolver un problema.

Al comenzar a abordar un problema hay que tener en cuenta que para la mayoría de ellos hay muchas maneras de resolverlos y pueden existir muchas soluciones, se deben explorar los criterios conceptuales de la obra y decidir la estrategia para su creación.

En lo que refiere a tiempo real, dicho término aplica a sistemas ideales, que, en sentido estricto, es inexistente; por lo que ningún modelo es una representación exacta de la realidad, su validez depende de lo razonables que sean las estimaciones o suposiciones tomadas y de su grado de aproximación a la realidad. Si se pretende generar obra evolutiva a partir de la variable de tiempo real como dato fijo, el resultado sólo puede ser un sistema que contabiliza tiempo con precisión.

El desarrollo tecnológico ha impulsado (e impulsa) la aparición de nuevas formas de expresión, las herramientas por sí mismas no representan un estímulo para la 
creatividad personal, pues la capacidad artística depende de factores más profundos que la mera disposición de medios tecnológicos.

Para producir obra artística por medio de tecnologías e implementar el uso de algoritmos, es necesario hacer el desarrollo conceptual de la obra y proponer una hipótesis que permita visualizar de forma esquemática los momentos o etapas que se deben resolver por medio de uno o varios algoritmos. Un ejemplo de desarrollo escultórico, aplicando modelado basado en algoritmos y sucesión Fibonacci se muestra a continuación (ver Fig. 9 y 10) [22].

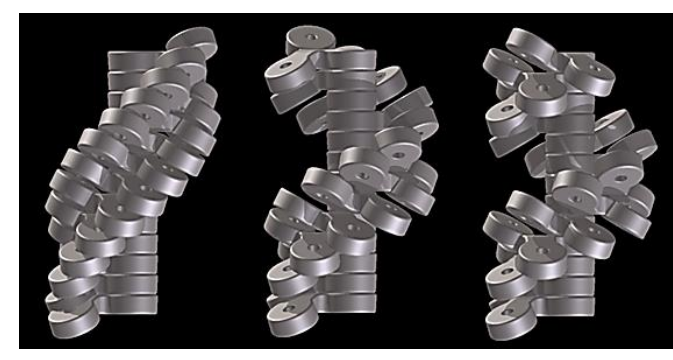

Fig. 9. Escultura modular con un desarrollo basado en un acomodo de orden radial con un número de módulos correspondiente a la sucesión de Fibonacci y un ángulo de separación variable.

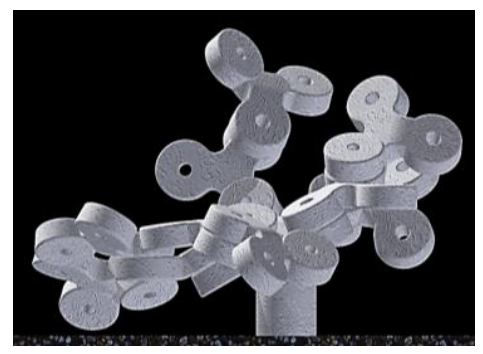

Fig. 10. Escultura modular con un desarrollo basado en un acomodo de orden programado en sucesión de Fibonacci y número de módulos con base en un algoritmo de equilibrio.

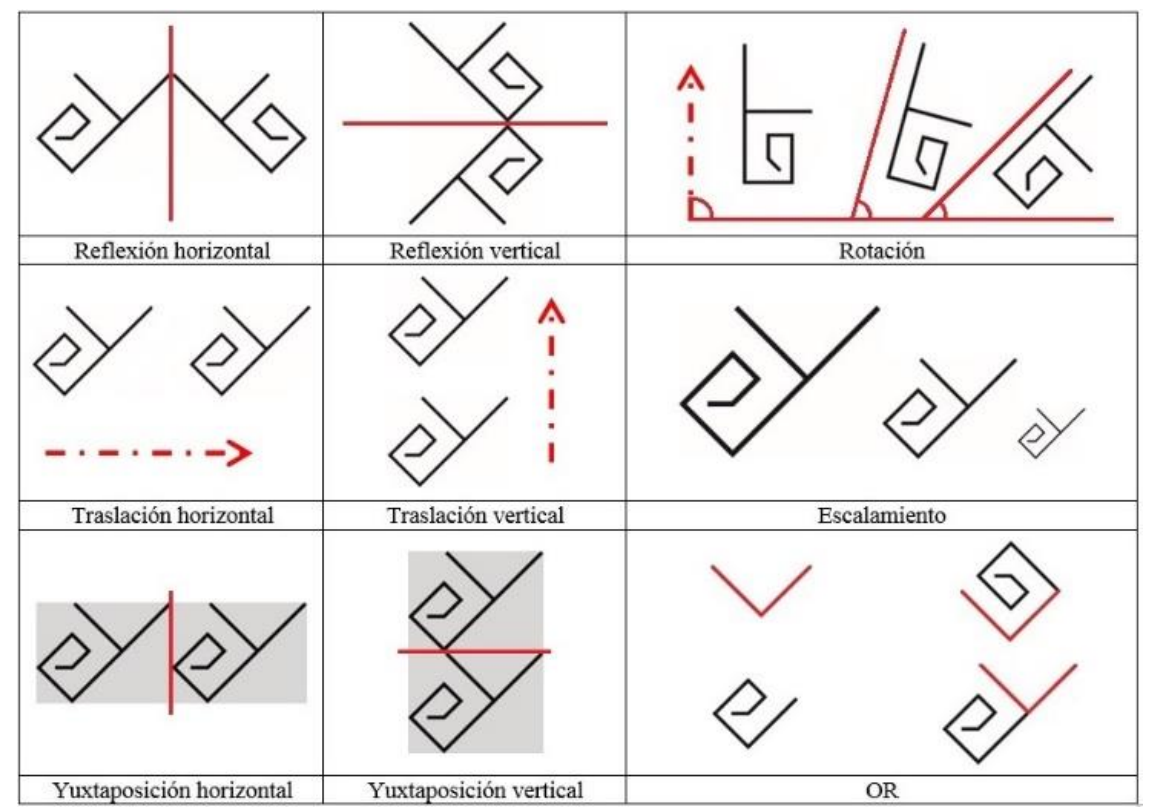

Fig. 11. Representación visual de operadores. 
Otro ejemplo de desarrollo modular basado en un programa de reconocimiento de patrones se muestra a continuación, donde dicho reconocimiento de patrones opera por medio de un autómata de pila que procesa íconos para obtener imágenes de indumentaria indígena por medio de un algoritmo de evaluación de cadenas en notación postfija. Estos desarrollos se basan en modelos formales de iconografía de textil indígena de la región de los Altos de Chiapas, Chiapas, México. Así, la aplicación de operadores a los íconos ha permitido reconstruir una imagen original (ver Fig. $11 \mathrm{y}$ 12) $[23]$.
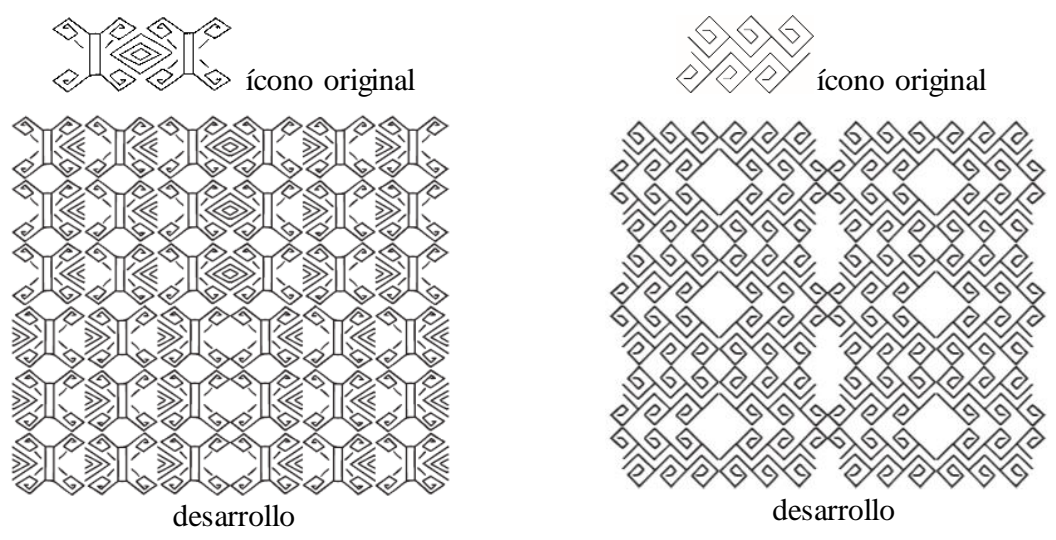

Fig. 12. Desarrollos visuales obtenidos por medio de aplicación de operadores en un autómata de pila que opera por medio de un algoritmo de evaluación de cadenas en notación postfija.

Obra de artistas

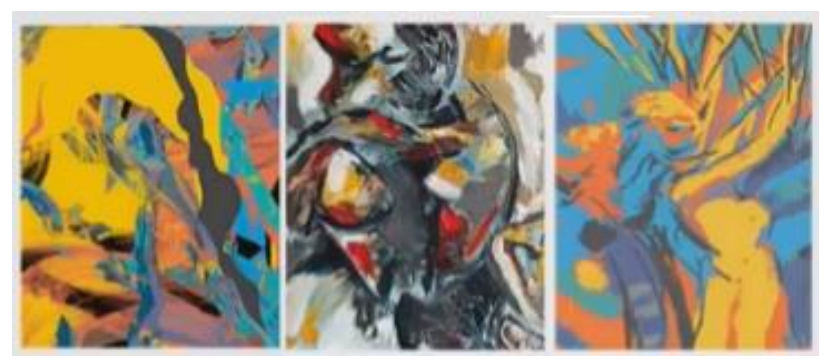

Obra de IA

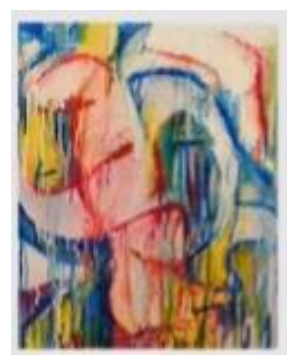

Fig. 13. Comparativo de obra artística producida por artistas e IA [23].

Finalmente, y como un ejemplo reciente, pero con base de desarrollo similar a los casos antes mencionados, Francisco Ordóñez [24], en su conferencia "¿Sueñan los androides con obras de Banksy? [...]", nos hace una reseña de algunas aplicaciones de Inteligencia Artificial (AI) para la generación de expresiones artísticas en diversos campos, entre ellos la de obra pictórica producto de algoritmos y redes neuronales con bancos de datos visuales tales como fotografías de cuadros de artistas plásticos, lo que 
ha permitido generar patrones de cualidades formales de la obra analizada con el fin de producir obra original aplicando estas cualidades.

En esta conferencia, para demostrar su premisa, Ordóñez somete a evaluación de la audiencia un grupo de imágenes para identificar cual es producto de IA, sin embargo, se precisa de un anális is que permita demostrar el proceso para identificar la obra de IA y comprobar la hipótes is (ver Fig. 13).

Cabe resaltar que la IA porsi sola tiene la capacidad de producir propuestas visuales con mayor velocidad que el ser humano, pero ello no garantiza la creación de conceptos o estilos para la producción artística, aunque si es una poderosa herramienta que potencializa capacidad productiva de un artista y hasta el momento depende del desarrollador del concepto, que a su vez debe identificar qué procesos de la producción artística se pueden mejorar por medio IA.

\section{Referencias}

1. Domínguez Bedoya, J.: El concepto de obra de arte en la legislación mexicana. Alegatos, noviembre 2010, núm. 11 pp. 1-7, www.azc.uam.mx/publicaciones/alegatos/pdfs/11/11-03. Último acceso: 26/03/19 (2010)

2. Padilla Padilla, R.A.: La obra plástica y su protección en la legislación panameña. http://www.legalinfo-panama.com/articulos/articulos_29a.htm, Último acceso: 20/02/19 (2003)

3. Fernández Martínez, L.M.: Los retos sociales y comunicativos de la pintura mural santiaguera, en la formación del individuo. Revista caribeña de ciencias sociales (2013)

4. Smith, T.: ¿Qué es el arte contemporáneo? Siglo XXI, México, p.18 (2012)

5. Regil Vargas, L.: Hipermedia: medio, lenguaje herramienta del arte digital. Revista Digital Universitaria 6(10), 2-18 (2005)

6. Escobar Rodríguez, I.L.: Las bellas artes, http://plasticas.dgenp.unam.mx/ inicio/introduccion/bellas-artes, Último acceso: 26/03/19 (2009)

7. Zalaya Báez, R.: Tesis doctoral: Escultura matemática "Antecedentes en la historia del arte, desarrollo, perspectivas de evolución y clasificación por conceptos matemáticos". Universidad Politécnica de Valencia, España, pp. 152-163 (2005)

8. López García, J.C.: Algoritmos y programación. Fundación Gabriel Piedrahita Uribe, Colombia, pp. 96 (2009)

9. Pinales Delgado, F.J., Velázquez Amador, C.E.: Problemario de algoritmos resueltos con diagramas de flujo y pseudocódigo. Universidad Autónoma de Aguascalientes, México, pp. $172(2014)$

10. López, G., Jeder, I., Vega, A.: Análisis y Diseño de Algoritmos. Implementación en C y Pascal. Alfaomega, Argentina, pp. 336 (2009)

11. García, M.E.: Introducción a la informática. http://www.profmatiasgarcia.com.ar/uploads/tutoriales/Ej_resueltos_algoritmos.pdf, último acceso 05/03/19 (2013)

12. Ramírez, C.: Metodología de programación, programación en $\mathrm{C}$, aplicaciones electrónicas. https://www.academia.edu/14334848/algoritmos_y_programas, Último acceso: 05/03/19 (2011)

13. Veiga, L.A.: Tesis: Sistemas jerárquicos de tiempo real para adquisición de datos y control., http://sedici.unlp.edu.ar/bitstream/handle/10915/1350/Documento_completo.pdf?sequence =29, Último acceso: 13/04/19 (1999) 
14. Balderrama Vásquez, C.: Algoritmos. http://moodle2.unid.edu.mx/dts_cursos_mdl/ lic/IC/EA/AM/06/Algoritmos.pdf, Último acceso: 03/04/19 (2009)

15. Fogel, D.B.: What is evolutionary computation? Spectrum, IEEE, 37(2), 26, 28-32 (2000) \& Fogel, D. B.: Evolutionary Computation: Toward a New Philosophy of Machine Intelligence. IEEE Press, Piscataway, NJ. Third Edition (2006) http://www.galeon.com/dantethedestroy er/algoritmos.pdf, último acceso 15/04/19 (2010)

16. Goldberg, D.E: Genetic Algorithms in Search, Optimization and Machine Learning. Addison-Wesley Longman Publishing Co., Inc., Boston, MA, USA (1989)

17. Darwin, Ch: Descent of Man: Nuvision Publications (2007)

18. Gonzalo García, M.: Apuntes de: Teoría de Autómatas y Lenguajes Formales, ciencias.uis.edu.co/lenguajes/doc/chomsky.doc, último acceso 10/04/19 (2002)

19. Reyes Gómez, D.A.: Descripción y aplicaciones de los autómatas celulares. Universidad Autónoma de Puebla, Puebla, México, http://delta.cs.cinvestav.mx/ mcintosh/ cellularautomata/Summer_Research_files/Arti_Ver_Inv_2011_DARG.pdf. Último acceso: 14/04/19 (2011)

20. Prins, G.-J., van Koolwijk, B.: Synchronator, https://lullcec.org/2010/concerts/sy nchronatorgert-jan-prins-bas-van-koolwijk/. Último acceso: 14/04/19 (2010)

21. Pighin, S.: Informática Aplicada a la Ingeniería de Procesos I (Orientación I). Universidad Tecnológica Nacional, Facultad Regional, Rosario, Argentina, https://www.frro.utn.edu.ar/repositorio/catedras/quimica/5_anio/orientadora1/monograias/p ighin-datamining.pdf. Último acceso: 14/04/19 (2001)

22. Rodríguez Mondragón, S.: Tesis: Sistema modular para conformación de escultura cerámica monumental. UAM, México (2014)

23. Rodríguez, S., Soto, L.J., Herrera, O.: Modelo de proceso para identificación visual a partir de íconos. EAE (2018)

24. Ordóñez Morales, F.J.: ¿Sueñan los androides con obras de Banksy? Creatividad, arte e IA. En: T3chFest 2019, Universidad Carlos III de Madrid, España, https://www.youtube.com/watch?v=gNkjJD1JSbw\&feature=share, Último acceso: 7/05/19, (2019) 